\title{
Ising Like Order by Disorder In The Pyrochlore Antiferromagnet with Dzyaloshinskii-Moriya Interactions
}

\author{
B. Canals, M. Elhajal, C. Lacroix \\ ${ }^{1}$ Institut NEEL, CNRS \& Université Joseph Fourier, \\ BP 166, F-38042 Grenoble Cedex 9
}

\begin{abstract}
It is shown that the mechanism of order out of disorder is at work in the antisymmetric pyrochlore antiferromagnet. Quantum as well as thermal fluctuations break the continuous degeneracy of the classical ground state manifold and reduce its symmetry to $\mathbb{Z}_{3} \times \mathbb{Z}_{2}$. The role of anisotropic symmetric exchange is also investigated and we conclude that this discrete like ordering is robust with respect to these second order like interactions. The antisymmetric pyrochlore antiferromagnet is therefore expected to order at low temperatures, whatever the symmetry type of its interactions, in both the classical and semi classical limits.
\end{abstract}

\section{INTRODUCTION}

The role of geometrical frustration in magnetic systems is at present one of the open question in the physics of strongly correlated systems since it can lead to novel low temperature behaviors. One of the most studied model is the nearest neighbor antiferromagnetic Heisenberg model on the pyrochlore lattice. For classical spins, the ground state is known to have macroscopic degeneracy, preventing any magnetic ordering at $\mathrm{T}=0$. In real systems, this degeneracy is often removed, partially or totally, by additional interactions such as dipolar interactions $\stackrel{1}{ }$, 2nd neighbor exchange ${ }^{2}$, single ion anisotropy ${ }^{3}$, magnetoelastic coupling ${ }^{4} \ldots$ This explains why pyrochlore compounds often order at low temperature, but their ordering temperature is usually much smaller than the paramagnetic Curie temperature $\Theta_{p}$ since $T_{N}$ is not related to the exchange interaction $\mathrm{J}$, but to the additional small interaction. Another type of process which can remove the degeneracy is order by disorder: as proposed first by Villain ${ }^{\underline{5}}$ the classical degeneracy can be lifted by fluctuations, if fluctuations around these classical ground states select one of these states. Both classical and quantum fluctuations are able to produce order by disorder, trough their contribution to entropy or zero point energy respectively. In this paper we study the effect of DzyaloshinskyMoriya interactions in the Heisenberg pyrochlore model. In an earlier paper $\stackrel{11}{ }$, we have shown that the $\mathrm{T}=0 \mathrm{~K}$ degeneracy is completly removed only for one particular choice of the DM vectors. Here we show that if the $\mathrm{DM}$ vectors are such that the $\mathrm{T}=0$ degeneracy is not removed, both classical and quantum fluctuations select 6 equivalent ordered configurations,leading to a well defined magnetic structure.

\section{DMI AND MORIYA'S RULES FOR THE PYROCHLORE LATTICE}

Dzyaloshinsky has shown ${ }^{6}$ that, in crystals with no inversion center, the usual isotropic exchange $J \vec{S}_{i} \cdot \overrightarrow{S_{j}}$ is not the only magnetic interaction and antisymmet-

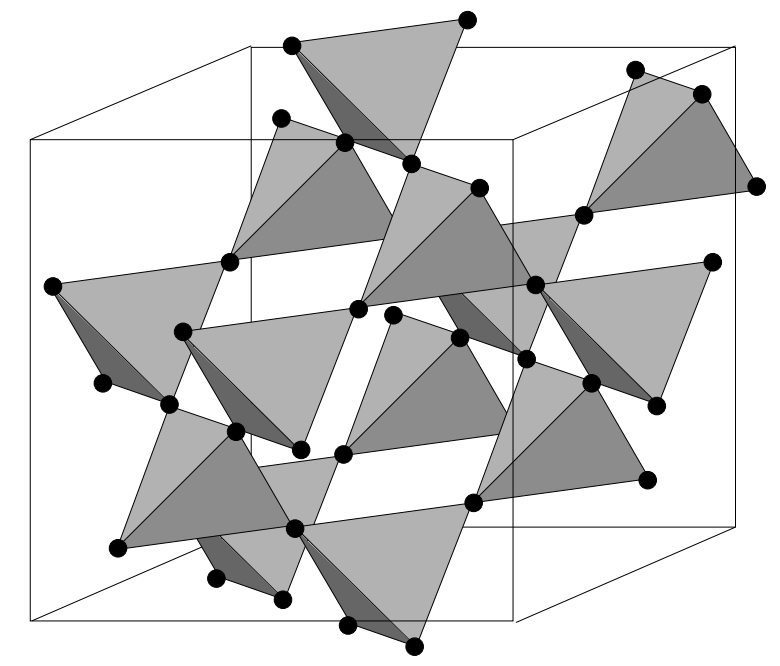

FIG. 1: The pyrochlore lattice. The interplay of the frustration of the tetrahedral unit cell with the weak connectivity of the (corner sharing) tetrahedra provides peculiar magnetic properties to the pyrochlore antiferromagnet.

ric exchange $\vec{D}_{i j} \cdot\left(\vec{S}_{i} \times \vec{S}_{j}\right)$ is allowed. In magnetic oxydes, exchange interactions are usually attributed to superexchange mechanism, which involves virtual hopping between 2 neighboring magnetic ions. Taking into account Coulomb repulsion and Pauli's principle,Anderson has explicited a microscopic mecanism which leads to isotropic super-exchange interactions. Later, Moriya has shown ${ }^{7.8}$ that inclusion of spin-orbit coupling on the magnetic ions in 1st and 2nd order leads to antisymmetric exchange and anisotropic exchange respectively. Moriya's microscopic derivation of the DMI is only valid for insulators but other possible microscopic mecanism relevant for other materials were explicited, for instance in systems with RKKY interactions $\mathbf{9}, 10$.

Whatever the microscopic origin of the DMI is, there are always symmetry constraints on the possible $\mathbf{D}$ vectors which may appear in the hamiltonian. Indeed, the hamiltonian must be invariant under the symmetry operations of the crystal and this will restrict the possible 

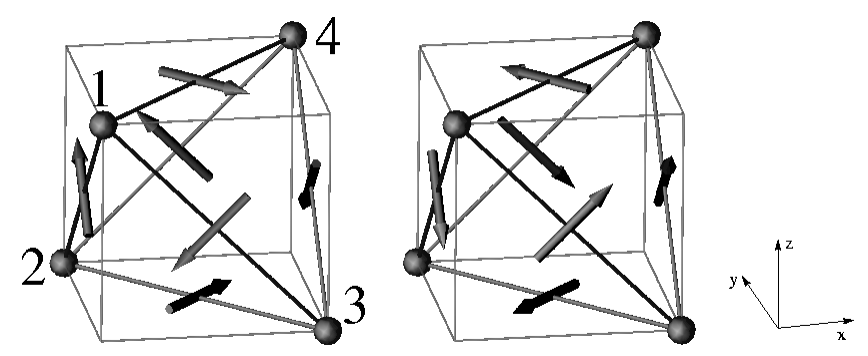

FIG. 2: D vectors for the DMI in the pyrochlore lattice. The convention is taken to fix the order for the cross products (always D. $\mathbf{S}_{i} \times \mathbf{S}_{j}$ with $j>i$ ). The two possible DMI are those obtained by varying the direction of the $\mathbf{D}$ vectors $(\mathbf{D} \rightarrow-\mathbf{D})$. The case with the $\mathbf{D}$ as represented on the left is refered to as the "direct" case and the other case (on the right) is the "indirect" case. Once the DMI between two spins is fully specified, the others DMI are also fixed and obtained by applying the different $\frac{2 \pi}{3}$ rotations around the cube's diagonals which leave the tetrahedron invariant. The DMI in the rest of the lattice are also fixed and obtained by applying appropriate symmetry operations of the pyrochlore lattice.

D vectors to those for which $\vec{D}_{i j} \cdot(\overrightarrow{S i} \times \overrightarrow{S j})$ is invariant under these symmetry operations. This way of constraining the $\mathbf{D}$ vectors has been given the name of Moriya's rules. Using these rules, we have shown $\underline{11}$ that two types of DM's interactions are compatible with the pyrochlore space group symmetry. These two patterns of $\mathbf{D}$ vectors have been given the name of "direct" and "indirect" case and are described in figure 2 .

As shown in our previous work, the classical ground state is always $q=0$. It is non degenerate in the direct case and is described by the so called all-in all-out magnetic phase; this case will not be discussed in this paper. From now on, we will focus on the second case of indirect DMI, where the ground state manifold is reduced compared to the case with no DMI, but is still continuously degenerate as shown in Ref. 11. The role of anisotropic exchange will also be discussed in this paper.

\section{ROLE OF FLUCTUATIONS WITHIN THE GROUND STATE MANIFOLD}

From now on, we will focus on the second case of indirect DMI, where the ground state manifold is $q=0$, but is continuously degenerate. There are two kinds of ground states 11 : (i) phases can be coplanar, belonging either to the $x y, y z$ or $z x$ plane and consists in perpendicular pairs of anticollinear spins (see figure 3) which can rotate freely within these planes. (ii) There are also non

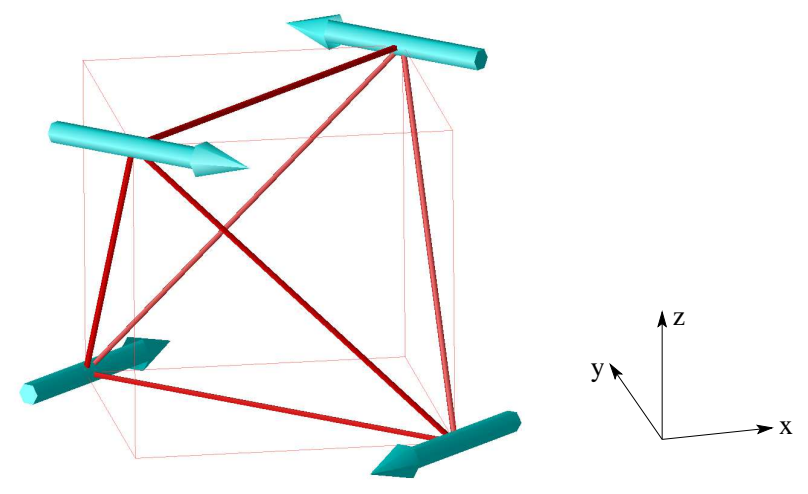

FIG. 3: One possible ground state in the case of indirect DMI. The ground state for the whole pyrochlore lattice is a $\mathbf{q}=\mathbf{0}$ structure so that only one tetrahedron is represented. Similar structures in the $z x$ and $y z$ planes are degenerate.

coplanar phases, parametrized by one angle $\varphi$ as follow,

$$
\begin{gathered}
\mathbf{S}_{1}=\left\{\begin{array}{l}
\cos \theta \cos \left(\varphi-\frac{\pi}{4}\right) \\
\cos \theta \sin \left(\varphi-\frac{\pi}{4}\right) \\
\sin (\theta)
\end{array} \quad \mathbf{S}_{2}=\left\{\begin{array}{l}
\cos \theta \cos \left(-\varphi+\frac{\pi}{4}\right) \\
\cos \theta \sin \left(-\varphi+\frac{\pi}{4}\right) \\
-\sin (\theta)
\end{array}\right.\right. \\
\mathbf{S}_{3}=\left\{\begin{array}{l}
\cos \theta \cos \left(-\varphi-\frac{3 \pi}{4}\right) \\
\cos \theta \sin \left(-\varphi-\frac{3 \pi}{4}\right) \\
-\sin (\theta)
\end{array} \mathbf{S}_{4}=\left\{\begin{array}{l}
\cos \theta \cos \left(\varphi+\frac{3 \pi}{4}\right) \\
\cos \theta \sin \left(\varphi+\frac{3 \pi}{4}\right) \\
\sin (\theta)
\end{array}\right.\right.
\end{gathered}
$$

where the spins are labeled as in figure 2 and where $\varphi$ and $\theta$ are not independant :

$$
\theta=\arctan (\sqrt{2} \sin \varphi)
$$

Consequently, it is possible to picture the classical ground state manifold by following the paths defined by the arrow of say, spin 1, in all allowed configurations (actually, fixing any spin determines the configuration of the 3 other spins). Such paths are depicted in figure 4 .

The ground state is therefore a multiply arcwise connected one dimensional manifold, represented by the thick black lines on figure 4. Six configurations play a particular role, since they are at the intersection of two lines, i.e they belong to both kinds of ground states defined above, where spins are coplanar, either in the $x y, y z$ or $z x$ plane (see Fig. (3)). In these phases, take for instance the $x y$ plane, two spins are anti parallel (aligned along the (110) axis) and perpendicular to the two remaining spins (aligned along the (110) axis). These configurations, related by time reversal symmetry and rotations, are globally invariant under $\mathbb{Z}_{3} \times \mathbb{Z}_{2}$, and will be referred to this way from now on.

It is of practical and theoretical interest to test wether fluctuations operate on that manifold and reduce the degeneracy. Before getting into a quantitative analysis, it is worth pointing out qualitative arguments towards an 


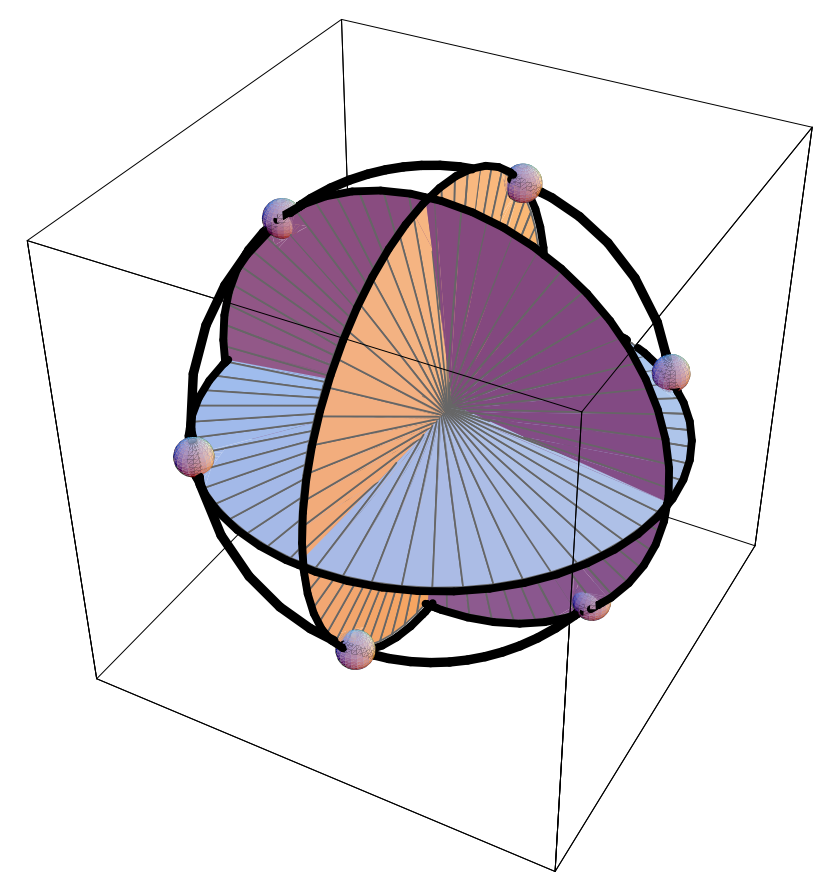

FIG. 4: Description of the one dimensional ground state manifold. Any move within the ground state can be described by the possible path followed by only one of the four spin as they are all parametrized by the same angle (see Eq.11). Here, only spin 1 is described. It can belong either to the $x y, y z$ or $z x$ plane, or be in a non coplanar phase. These non coplanar phases intersect coplanar phases at 6 points and the only way to go from one plane to another is to pass through these intersections, depicted by spheres.

order out of disorder mechanism. The main ingredient for fluctuations to be efficient resides in the spectrum of the system : if two degenerate phases have different spectra and in particular, if one has softer modes, it is common that this one is selected at low temperatures, where entropic effects and/or quantum fluctuations may take place. The measure of the ground state manifold also plays an important role and should be sufficiently small. Schematically, if one phase is to be selected by fluctuations but is "drowned" in a huge ground state manifold, the system cannot statistically pick up that phase and fluctuations will therefore not operate. In the present case, as it is shown hereafter, both preceding conditions are fulfilled : there are obviously peculiar points expected to have spectra with softer modes (zero modes actually) and the ground state manifold, because it is one dimensional, has a null measure in configuration space and allows order out of disorder to be efficient, both at thermal and quantum levels.

\section{A. Thermal fluctuations}

As emphasized in the previous paragraph we can guess that the peculiar points of the configuration space around which thermal fluctuations could be efficient are the above mentioned 6 points because they are the only one within the ground state manifold having two obvious "escape lines" e.g, two zero energy modes. It is therefore natural to define a parameter which is maximum and equal to one only when spin configurations are among the previous six. In each of these configurations, spins all belong to the same plane, either $x y, y z$ or $z x$. When they belong to the $x y$ plane, spins 1 and 4 are collinear as are spins 2 and 3 , with the 1-4 pair being perpendicular to the 2-3 pair (see figure 3). The same occurs for the two other planes. Therefore, for each plane selection, a global index $\alpha$ can be defined to label a vector $e_{i}^{\alpha}$ on each site of the lattice, so that the quantity

$$
m_{\alpha}=\frac{1}{N} \sum_{i}^{N} \vec{S}_{i} \cdot \vec{e}_{\alpha}
$$

equals \pm 1 for the two time reversal related phases of each plane selection $\alpha=x y, y z, z x$. If the spins belong to the $x y$ plane, they point along the $(1,-1,0)$ direction, thus $e_{x y}=\frac{1}{\sqrt{2}}(1,-1,0)$ and similarly for the two other planes. Defining then

$$
m=\max _{\alpha}\left(m_{\alpha}^{2}\right)
$$

allows for characterizing any spin configuration and its proximity to one of the six peculiar phases because $M=$ 1 for these configurations and these configurations only.

In order to test wether thermal fluctuations may entropically select peculiar phases of the ground state manifold, we have performed classical monte carlo simulations of finite size lattices with periodic boundary conditions. The clusters we investigated had 32, 500 and 2048 sites. For each simulations, we used a single flip metropolis algorithm with a modified update so that the acceptance rate stayed roughly above $40 \%$, associated to a local rotation of each spin around its local molecular field. During the simulation, the autocorrelation time is calculated on the fly in order to adapt the number of monte carlo steps between two measures and ensure that measures are uncorrelated. Results are reported in Fig 5 and 6 for respectively $D / J=0.1$ and $D / J=1$. The measure of $\langle m\rangle$ as a function of the temperature clearly indicates that this parameter is an order parameter, although we cannot discuss the order of the transition so far.

Both cases support that at low temperature, the system orders in one of the 6 previously described states as was expected from qualitative arguments. Moreover, it can be seen that $T_{C}$ is of the order of $D$, which confirms that DMI are responsible for this ordering. 


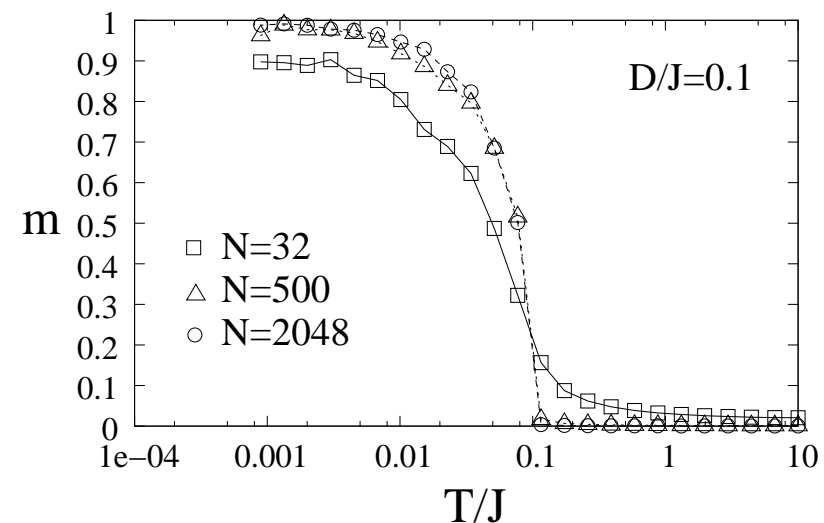

FIG. 5: Thermal average of $m$ (see Eq. 31) as a function of temperature for three lattice sizes, $N=32,500$ and 2048, and for $D / J=0.1$.

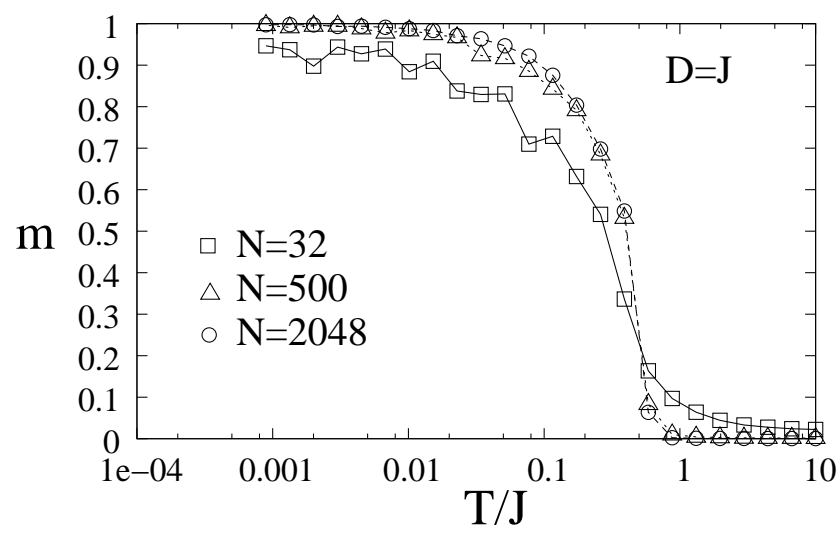

FIG. 6: Thermal average of $\mathrm{M}$ (see Eq. 3) as a function of temperature for three lattice sizes, $N=32,500$ and 2048, and for $D / J=1.0$.

\section{B. Quantum fluctuations : semiclassical approach}

The same investigation has been done for quantum fluctuations. Contrary to the classical case, it is not necessary to guess which points will play a particular role. This is because it is possible to compute at zero temperature the energy renormalized by quantum fluctuations for all phases of the ground state manifold. To do so, we have performed a linear spin wave expansion of the Hamiltonian around each state of the ground state manifold, e.g, around each state parametrized by the angle $\varphi$. The resulting bosonic hamiltonian is then diagonalized which allows to obtain the normal modes and calculate the renormalized energy and magnetization by quantum fluctuations.

The first result is that the $\mathbb{Z}_{3} \times \mathbb{Z}_{2}$ phases are unambiguously selected by quantum fluctuations, as shown in Fig. 7. For planar phases, they correspond to $\alpha=0$ or

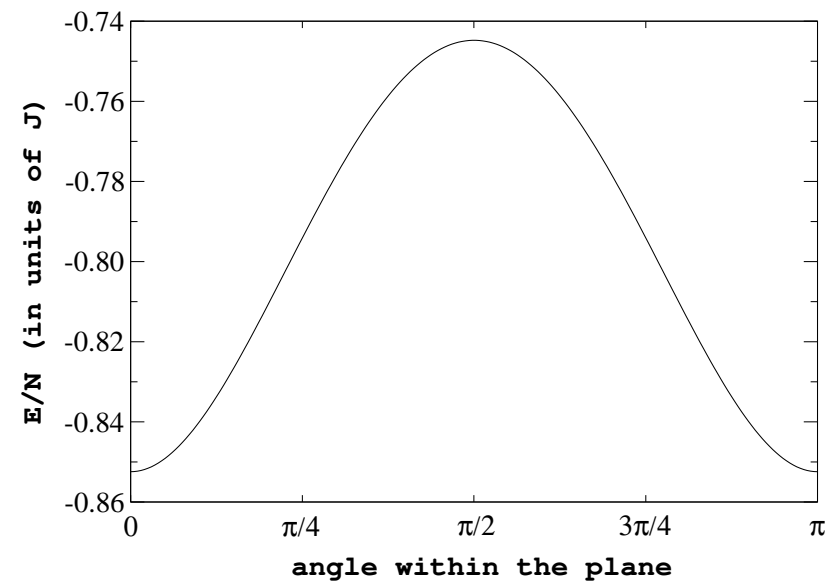

FIG. 7: Renormalized energy per site as a function of the angle $\alpha$, for planar phases [all planes are equivalent], with $D / J=1 . \alpha=0$ and $\alpha=\pi$ correspond to one time reversal $\mathrm{r}$

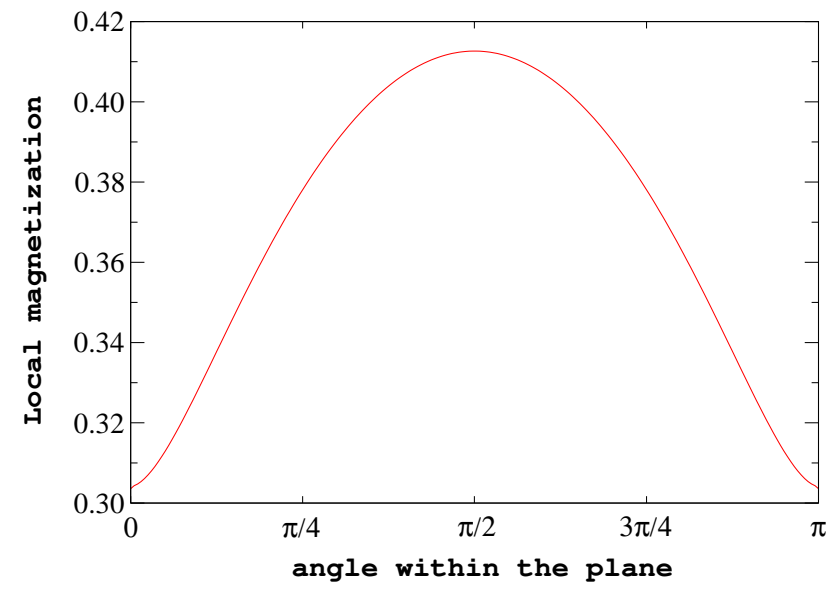

FIG. 8: Renormalized magnetization for $S=1 / 2$ as a function of the angle $\alpha$, for planar phases, with $D / J=1$. $\alpha=0$ and $\alpha=\pi$ correspond to one time reversal related pair of states belonging to the $\mathbb{Z}_{3} \times \mathbb{Z}_{2}$ phases. At these two points, fluctuations have softer spectra, hence local magnetization is much more renormalized.

$\alpha=\pi, \alpha$ being the angle of the spins with one of the 6 peculiar phases ( 2 for each plane). The same calculation was performed for conic phases (indexed by $\varphi$ ), confirming that the 6 phases are selected by quantum fluctuations.

This selection is also illustrated by the computation of the renormalized magnetization, as shown in Fig. 8 . As confirmed by the calculation of the energy, there are softer modes near the 6 selected phases. Consequently, transverse fluctuations are much stronger giving a larger renormalization of magnetization around these phases.

Wether the magnetization, which is zero for $D / J=0$, could be stabilized by taking DM's interactions into ac- 


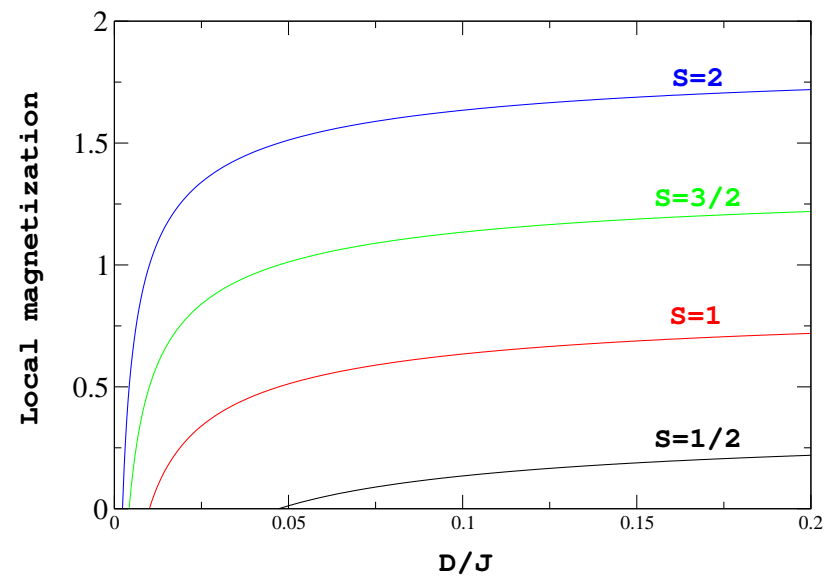

FIG. 9: Renormalized magnetization of one of the symmetry related $\mathbb{Z}_{3} \times \mathbb{Z}_{2}$ phases, as a function of $D / J$ for $S=1 / 2,1,3 / 2$ and 2. For $S \geq 1$, a DM interaction of 1 percent is sufficient to stabilize a Néel like long range order. From that point of view, DM interactions are very efficient in destroying the classical spin liquid behavior and driving this model to a classical behavior.

count is also of interest because it quantifies how easily this model becomes "classical". We have therefore computed for one of the six selected phases, the value of the quantum renormalized magnetization as a function of $D / J$. Results are reported in Fig. 9. It is worth noting that apart from the case $S=1 / 2$, a very small value of $D / J(D / J \gtrsim 0.01)$ stabilizes the magnetic ground state of the pyrochlore antiferromagnet. Furthermore, we can quantify how efficient DM's interactions are by fitting the gain in energy as a function of $D / J$. For small values of $D / J$, quantum fluctuations contribute to the renormalization of the energy as $\sqrt{D / J}$. This is an important point as discussed in the next section.

\section{ROLE OF ANISOTROPIC SYMMETRIC LIKE EXCHANGE}

So far, only DM's interactions have been taken into account. Whatever the type of fluctuations, thermal or quantum, the order out of disorder mechanism is efficient and reduces the continuous degeneracy to a discrete one. But is this selection robust vis à vis next order (in spin orbit coupling) interactions present in the Hamiltonian, the anisotropic symmetric exchange? Unfortunately, we must lose generality to address this question because many kind of symmetry allowed interactions are possible. It is nevertheless possible to argue quantitatively on that matter.

In order to be specific, we choose to consider interactions that keep all symmetries of the pure pyrochlore lattice. They correspond to nearest neighbour truncated dipolar interactions $\frac{12}{2}$,

$$
K \frac{\left(\hat{n}^{\alpha} \cdot R_{i j}^{m n}\right)\left(\hat{n}^{\beta} \cdot R_{i j}^{m n}\right)}{\left|R_{i j}^{m n}\right|^{5}} .
$$

where indices $i, j$ are related to the fcc Bravais lattice while indices $m, n$ correspond to one of the four sites of the tetrahedral unit cell. The set of unit vectors $\hat{n}^{\alpha}$ is $(1,0,0),(0,1,0),(0,0,1)$. We allow the sign of $K$ to vary as there is no obvious reason why one peculiar type, $K>0$ or $K<0$, should prevail. The full hamiltonian we are left with reads now,

$$
\mathcal{H}=-\frac{1}{2} \sum_{i, j} \sum_{m, n} \sum_{\alpha, \beta} \mathcal{J}\left(R_{i j}^{m n}\right)^{\alpha \beta} S_{i}^{m, \alpha} S_{j}^{n, \beta}
$$

where $\mathcal{J}\left(R_{i j}^{m n}\right)^{\alpha \beta}$ are the coefficients of the coupling matrix $\mathcal{J}(\boldsymbol{q})$ which contains the isotropic symmetric exchange $-J S_{i} . S_{j}$, the antisymmetric exchange $D_{i j} . S_{i} \times$ $S_{j}$ and the anisotropic symmetric exchange $K$ (see Eq. (4). Stabilized low temperature phases are investigated through a mean field analysis. The hamiltonian is rewritten in reciprocal space using the following transformations,

$$
\begin{aligned}
S_{i}^{m, \alpha} & =\frac{1}{\sqrt{N}} \sum_{\boldsymbol{q}} S_{\boldsymbol{q}}^{m, \alpha} e^{-\imath \boldsymbol{q} \cdot \boldsymbol{R}_{i}^{m}}, \\
\mathcal{J}\left(R_{i j}^{m n}\right)^{\alpha \beta} & =\frac{1}{N} \sum_{\boldsymbol{q}} \mathcal{J}_{m n}^{\alpha \beta}(\boldsymbol{q}) e^{\imath \boldsymbol{q} \cdot \boldsymbol{R}_{i j}^{m n}},
\end{aligned}
$$

where $N$ is the number of Bravais lattice points. The resulting interaction matrix $\mathcal{J}(\boldsymbol{q})$ is a $12 \times 12$ non diagonal hermitian matrix. Hence, to completely diagonalize $\mathcal{J}(\boldsymbol{q})$ one must transform the $\boldsymbol{q}$-dependent variables, $\boldsymbol{S}_{\boldsymbol{q}}^{m}$, to normal mode variables. In component form, the normal mode transformation is given by

$$
S_{\boldsymbol{q}}^{n, \alpha}=\sum_{p=1}^{4} \sum_{\gamma=1}^{3} U_{n, p}^{\alpha, \gamma}(\boldsymbol{q}) \phi_{\boldsymbol{q}}^{p, \gamma},
$$

where the indices $(p, \gamma)$ label the normal modes (12 for Heisenberg spins), and $\left\{\phi_{\boldsymbol{q}}^{p, \gamma}\right\}$ are the amplitudes of these normal modes. $U(\boldsymbol{q})$ is the unitary matrix that diagonalizes $\mathcal{J}(\boldsymbol{q})$ with eigenvalues $\lambda(\boldsymbol{q})$. Finally, the mean-field free energy to quadratic order in the normal modes reads, up to an irrelevant constant ${ }^{13}$,

$$
\mathcal{F}(T)=\frac{1}{2} \sum_{\boldsymbol{q}, p, \gamma}\left(n T-\lambda_{p}^{\gamma}(\boldsymbol{q})\right)\left|\phi_{\boldsymbol{q}}^{p, \gamma}\right|^{2},
$$

where $\mathcal{F}(T)$ is the mean-field free energy per unit cell, $T$ is the temperature in units of $k_{\mathrm{B}}$, and $n=3$ for Heisenberg spins. Therefore, the mean-field low temperature phase is defined by the corresponding wave vector $\boldsymbol{q}_{\text {ord }}$ associated with the extremal eigenvalue $\max _{p, \gamma, \boldsymbol{q}}\left(\lambda_{p}^{\gamma}(\boldsymbol{q})\right)$.

When $K=0$, it was shown in preceding sections that $\boldsymbol{q}_{\text {ord }}=0$ and that the ground state manifold is continuously degenerate. Including non zero $K$, whatever 
its sign, the degeneracy is lifted already at the mean field level. The new ground states are, for both positive and negative $K, \boldsymbol{q}=0$ slightly distorted version of the $\mathbb{Z}_{3} \times \mathbb{Z}_{2}$ states. The energy decrease, for small $K$, is always quadratic, $\Delta E \propto K^{2}$.

At this point, we can discuss wether anisotropic symmetric interactions may interfere with quantum fluctuations induced by DM's interactions. The first remark is that at the mean field level, $K$ breaks the degeneracy but select phases which are continuous deformation of quantum selected phase. Therefore, we expect that taking into account anisotropic exchange would not change the behavior of this system. The second point is related to how strong $K$ is efficient in selecting a particular phase. Suppose that the mean field selected phase is not one of the $\mathbb{Z}_{3} \times \mathbb{Z}_{2}$ phases or a slightly distorted version of those. Because the energy gain is quadratic in $K$, it appears that quantum fluctuations are much more efficient, as they induce an energy gain proportional to $\sqrt{D}$ (see section IIIB). This clearly shows that even if anisotropic interactions are taken into account, we expect a lifting of degeneracy corresponding to the $\mathbb{Z}_{3} \times \mathbb{Z}_{2}$ phases or slightly distorted versions of those. The only mechanism which could work against such scenario would be that quantum fluctuations around $K$ selected phases be larger than $\sqrt{D}$. Because $K \propto D^{2}$, this would mean that one should expect the energy gain induced by $K$-like interactions to behave like $\sqrt[4]{K}$, which is highly unprobable.

\section{CONCLUSION}

This works investigates the role of thermal and quantal fluctuations on the antisymmetric pyrochlore antifer- romagnet. When the symmetry type of the allowed DMI leads to a one dimensional degenerate ground state, it is shown that these two types of fluctuations are efficient in reducing the degeneracy and drives the model to the same ordered ground state with a discrete global degeneracy of the $\mathbb{Z}_{3} \times \mathbb{Z}_{2}$ type. Including higher order terms in the hamiltonian like the anisotropic symmetric exchange is likely to leave thermal and quantal fluctuations dominate the low temperature behavior of this system. It is therefore concluded that whatever the type of symmetry allowed DMI, direct or indirect, they should drive the pyrochlore Heisenberg antiferromagnet to a low temperature ordered phase. When the model is essentially classical, the critical temperature is of order $\mathbf{D}$. If quantum fluctuations are present, the value of the ordering temperature results from a balance between the strength of the DMI and the magnitude of the fluctuations. As we have shown that for moderately large DMI the local magnetization is quite stable, the value of the ordering temperature should not be very different from the one of the classical case. Finally, it is worth pointing out that most of studied pyrochlore compounds are Rare Earth oxides. It is therefore the next natural step to investigate the mechanism of DMI within $5 f$ alloys and determine whether those interactions could be in some compounds large enough to drive the low temperature behavior of these frustrated systems.
1 S.E. Palmer and J.T. Chalker, Phys. Rev. B 62, 488 (2000)

2 J.N. Reimers, A.J. Berlinsky and A.C. Shi, Phys. Rev. B 45, 2899 (1992)

${ }^{3}$ M. J. Harris, S. T. Bramwell, D. F. McMorrow, T. Zeiske, and K. W. Godfrey, Phys. Rev. Lett. 79, 2554 (1997)

4 O. Tchernyshov, R. Moessner, S.L. Sondhi, Phys. Rev. Lett 88, 067203 (2002)

5 J. Villain, Z. Phys B 33, 31 (1979)

${ }^{6}$ I. Dzyaloshinky, J. Phys. Chem. Solids 4, 241 (1958)

7 T. Moriya, Phys. Rev. Lett. 4, 228 (1960)
8 T. Moriya, Phys. Rev. 120, 91 (1960)

9 A. Fert and P.M. Levy, Phys. Rev. Lett. 44, 1538 (1980)

10 P.M. Levy and A. Fert, Phys. Rev. B 23, 4667 (1981)

11 M. Elhajal, B. Canals, R. Sunyer, and C. Lacroix, Phys. Rev. B 71, 094420 (2005)

12 The symmetric part of the dipolar-like interaction can be absorbed in the usual symmetric exchange.

13 M. Enjalran and M. J. P. Gingras, Phys. Rev. B 70, 174426 (2004). 